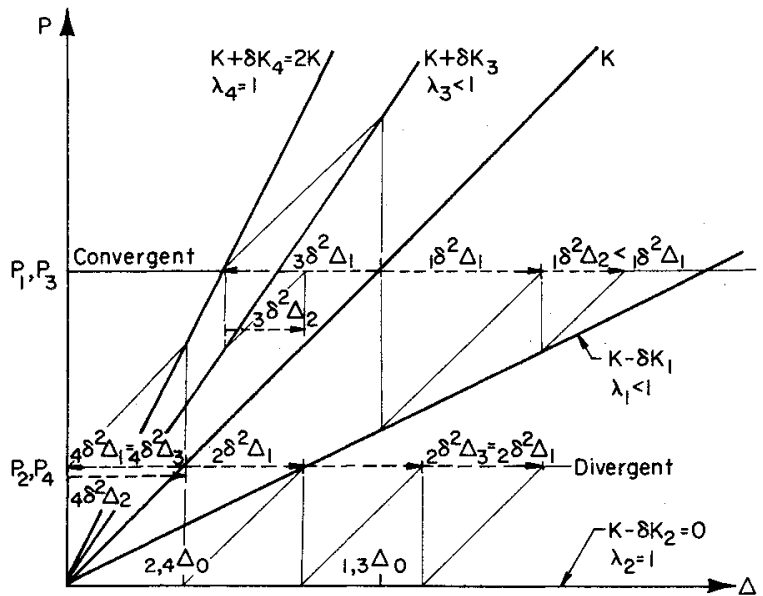

Fig. 2 Convergence modes $\left|{ }_{i} \delta^{2} \Delta_{n+1}\right|=\lambda_{i}\left|{ }_{i} \delta^{2} \Delta_{n}\right|$.

simultaneous satisfaction of two criteria is recommended for terminating the iteration cycles. One criteria is that the change in deflections between two iterations $\left(\delta^{2} \Delta_{(n)}\right)$, as measured by $E_{n}$, be sufficiently small. The other criteria is that the change in strain energy $(\delta U)$ between two iterations be sufficiently small as compared to the strain energy $(U)$ to assure that the stress changes are not of any engineering significance.

i.e. $E_{n} \leq$ TOL $1 \sim$ (maximum acceptable deflection error $)^{2}$ times the number of displacements

$$
\frac{\delta U}{U} \leq \text { TOL } 2 \sim \underset{\text { ratio })^{2}}{(\text { maximum acceptable stress error }}
$$

where

$$
\delta U=(P)^{T}\left(\delta^{2} \Delta\right)
$$

and

$$
U=(P)^{T}\left(\Delta_{0}+\delta \Delta\right)
$$

It should be noted that the recommended iterative scheme is even more efficient when the modification increases the stiffnesses of the members. In this case the geometric matrix series solution represents an alternating series. This requires a large number of linear (oscillating) iterations to approach the solution, while the geometric series extrapolation requires a much smaller number of trial solutions. The recommended geometric solution is

$$
\delta \Delta=\delta \Delta_{n}+\frac{\delta \Delta_{n+1}-\delta \Delta_{n}}{I-\left(\operatorname{sgn}\left[\delta^{2} \Delta_{n+l}\right]^{T}\left[\delta^{2} \Delta_{n}\right]\right)\left(E_{n+l} / E_{n}\right)^{1 / 2}}
$$

The iterative algorithm consists of selecting a good initial guess as to the change in displacement $\left(\delta \Delta_{n-1}\right)$ due to the structural modification $(\delta K)$. The first initial guess is $\delta \Delta_{0}=$ $A \Delta_{0}$. Equation (4a) is then employed to determine values of $\delta \Delta_{n}$ and $\delta \Delta_{n+1}$. The values of $\delta^{2} \Delta_{n}, \delta^{2} \Delta_{n+1}, E_{n}, E_{n+l}$, and $\lambda$ are also calculated from Eqs. (5a) and (5c) and substituted into Eq. (7) to determine a new initial guess. This computational scheme is repeated as many times as required. The decision as to the acceptable accuracy of the last initial guess is based upon satisfaction of Eq. (6). It should be noted that the iterative scheme does not require the inversion of the stiffness matrix $\left(K^{-I}\right)$. Any stiffness such as the main diagonal or upper triangle, which are easily inverted $(F)$, may be utilized provided the norm of $F \delta K$ is less than one.

The mode of convergence is schematically presented in Figs. 1 and 2. Figure 2 represents the Eq. (2d) solution of a one degree of freedom (invariant eigenvector) structure. Note that the value of $\delta^{2} \Delta_{n}$ always decrease whenever $\lambda=|\delta K / K|<1.0$, guaranteeing convergence. A reduction in stiffness results in positive $\delta^{2} \Delta_{n}$, while an increase in stiffness causes an oscillation in sign and a slower convergence. Both solutions would be accelerated with the algorithm. Values of $\lambda \geq 1.0$ result in nondecreasing values of $\delta^{2} \Delta_{n}$, indicating that Eqs. (2e) or (5b) should not be utilized for major increases of stiffness or an unstable structure. The increase of stiffness can be handled by an arbitrary magnification of the original stiffness $\left(K_{2}=\mu K_{I}\right)$, so as to result in $\lambda_{l 2}=\lambda_{11} / \mu<1.0$.

The algorithm assumes that the linear damaged structure has adequate strength to withstand the resulting calculated stresses, which are a known linear transformation of the displacements. If this is not justified, then the linear structure can be reanalyzed with a "pseudo load" (equal to the load capacity of the overstressed members, e.g., yield, buckling, fracture) and an incremental $\delta K$ representing the elastic stiffness of these members. The iterative algorithm applies to linear structure, but can be advantageously reiterated for nonlinear structures, provided that the effect of the nonlinearity upon the $\delta K$ can be evaluated in the manner indicated préviously.

Note that efficient iterative solutions of original analysis problems of large number of degrees of freedom can be obtained by employing the readily available inverses of the main diagonal, without or with the upper triangle, as the flexibility matrix $(F)$. The difference between these matrices and the total stiffness matrix $(K)$ can be set equal to the change in stiffness $(\delta K)$. The latter technique (Gauss-Siedel) is more efficient since the norm of the corresponding change matrix $(A)$ is smaller. Utilizing the modification represented by extrapolation to the geometric series approximation should significantly increase the efficiency of these oscillating iterative techniques. The geometric series algorithm can also be utilized to increase the efficiency for the determination of eigenvalues and eigenvectors by iterative techniques.

\section{Slow Ions in Plasma Wind Tunnels}

\author{
W. A. Oran* \\ NASA Marshall Space Flight Center, Huntsville, Ala.

$$
\text { U. Samir } \dagger
$$ \\ University of Michigan, Ann Arbor, Mich. \\ Tel Aviv University, Ramat Aviv, Israel \\ and \\ N. H. Stoneł \\ NASA Marshall Space Flight Center, Huntsville, Ala.
}

\section{Introduction}

$\mathbf{T}$ HE interaction between a satellite and its space environment, which created disturbed zones around the satellite, has been studied since the early 1960's. Numerous theoretical models which describe aspects of the interaction are now available (e.g., Refs. 1-3); although, the degree of applicability of these models is not always well understood.

Received February 2, 1976; revision received April 19, 1976. Index categories: Rarefied Flows; Plasma Dynamics and MHD; Research Facilities and Instrumentation.

*Research Physicist, Magnetospheric and Plasnia Physics Branch, Space Sciences Laboratory.

$\uparrow$ Research Scientist, Department of Atmospheric and Oceanic Sciences, University of Michigan; Associate Professor, Department of Geophysics and Planetary Science, Tel Aviv University.

$\ddagger$ Research Physicist, Magnetospheric and Plasma Physics Branch, Space Sciences Laboratory. 


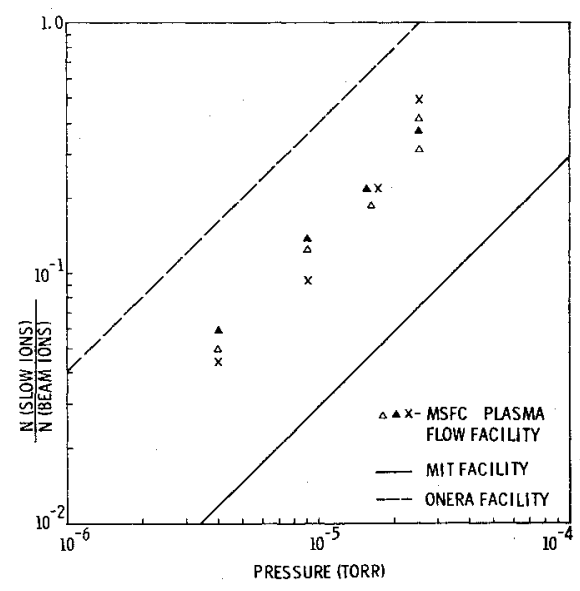

Fig. 1 Measurements of the ratio $(\alpha)$ of slow ion density to beam ion density versus pressure for the MFSC, MIT $^{8}$ and ONERA ${ }^{10}$ facilities. The data symbols mean: $x-\alpha$ determined from electron density variations in wake of body; $\Delta,-\alpha$ determined from ion current measurements in wake of body.

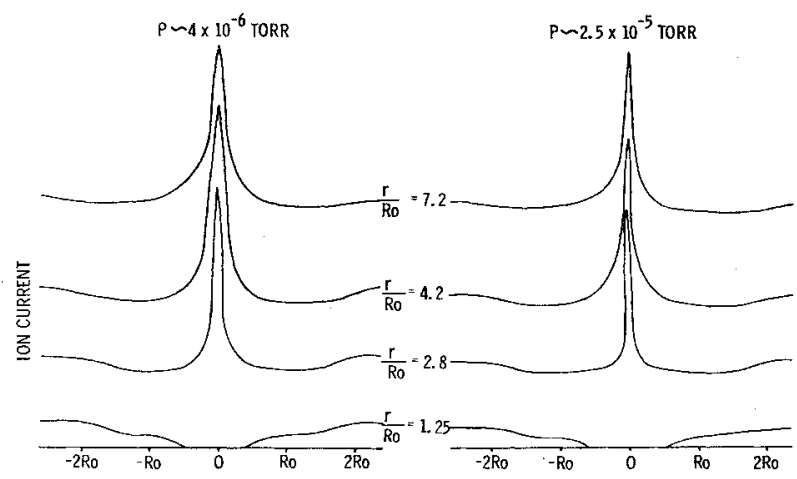

TRANSVERSE DISTANCE

Fig. 2 Transverse ion current profiles taken at different axial distances $r / R_{f}$ downstream from the center of a sphere (radius $=R_{0}=4$ $\mathrm{cm})$ at two different system pressures $p$ and at flow conditions of $E_{i}-4.5 \mathrm{eV}, T_{e}-800^{\circ} \mathrm{K}, n \sim 5 \times 10^{4} / \mathrm{cm}^{3}$ and $\phi_{s} \sim-2 \mathrm{~V}$.

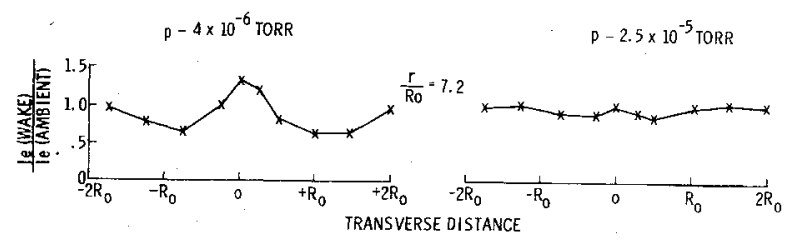

Fig. 3 Transverse electron current profiles (normalized to the ambient $I_{c}$ ) taken at an axial distance of $7.2 R_{j}$ downstream from the center of the sphere (radius $=R_{0}=4 \mathrm{~cm}$ ) at two different systems pressures $p$ and at flow conditions $E_{i} \sim 4.5 \mathrm{e}, \mathrm{V}, T_{e}$ $\sim 800 \mathrm{~K}, n-5 \times 10^{4} / \mathrm{cm}^{3}$ and $\phi_{s} \sim-2 \mathrm{~V}$.

Unfortunately, the available relevant in situ data, which could be used to evaluate the applicability of the various theories, is meager and fragmentary. ${ }^{4}$ However, laboratory studies which can partially simulate the interaction have been used to complement the in situ observations and have yielded a better understanding of the relative importance of the various plasma parameters such as $R_{0} / \lambda_{D}, e \phi / K T_{e}$, and $v_{s} /\left(2 K T_{e} / m_{i}\right)^{1 / 2}$ on the distribution of charged particles and potentials around bodies immersed in flowing plasmas (e.g., Refs. 5-7). (Note: $R_{0}=$ body radius, $\phi=$ body potential relative to plasma potential, $v_{s}=$ flow velocity, $\left(2 K T_{e} / m_{i}\right)^{1 / 2}$ $=$ "Mach" number, and the remainder of the symbols retain their standard meaning.)

One of the limitations of "simulation" experiments, which is often mentioned as being responsible for modifying the structure in the wake region, is the background of slow ions in the plasma chamber. These ions appear to be created by charge exchange between the beam ions and residual neutral gas and may affect measurements of the current and potential in the wake. In this Note, we will examine the influence of slow ions on both the ion and electron current distribution and the electron temperature in the wake of a body in a streaming plasma. The measurements were made in the Plasma Wind Tunnel Facility at NASA/MSFC, ${ }^{6}$ which is being used to partially simulate the wake structure of a satellite in the ionosphere.

\section{Experimental Results and Discussion}

The ratio of the slow ion density to the beam ion density $\alpha$ $=n$ (slow) $/ n$ (beam) was measured under a variety of plasma flow conditions. The experimental facility is described elsewhere. ${ }^{6}$ Of particular interest was the pressure range of from $\sim 10^{-6}$ to $\sim 10^{-5}$ Torr which comprises the nominal operating pressure of most plasma wind tunnels.

The slow ion density was determined from two independent measurements which are described in detail by Hester and Sonin ${ }^{8}$ and discussed by Martin and Cox. ${ }^{9}$ Figure 1 shows the ratio $\alpha=f$ (pressure) for the MSFC facility and compares it with conditions in the MIT facility ${ }^{8}$ and data from the ONERA facility. ${ }^{10}$ The MSFC data show that $\alpha$ is linearly proportional to the pressure in the chamber. This is in accordance with the behavior observed in the other two wind tunnels.

In Fig. 2 we present two typical sets of transverse ion current profiles taken at pressures of $4 \times 10^{-6}$ Torr and $2.5 \times 10^{-5}$ Torr at downstream distance of $r / R_{0}=1.25,2.8$, 4.2 , and 7.2 behind a sphere. The flow conditions were $E_{i} \sim$ $4.5 \mathrm{eV}, T_{e} \sim 800^{\circ} \mathrm{K}, n \sim 5 \times 10^{4} / \mathrm{cm}^{3}, \phi_{s}--2 \mathrm{~V}$, and $R_{0}=4$ $\mathrm{cm}$ where $E_{i}$ is the ion beam energy. The ion current was detected with a Faraday cup.

Figure 2 shows that the general structure of the ion current in the wake is similar in the pressure range of from $4 \times 10^{-6}$ Torr to $1.5 \times 10^{-5}$ Torr. This pressure range corresponds to $\alpha=n$ (slow) $/ n$ (beam) of $0.5 \leq \alpha \leq 0.3$ (see Fig. 1). The depleted region (which is sometimes called zone of maximum rarefaction) at $r / R_{0}=1.25$ is quantitatively similar at the two pressures. Ion current enhancements can be clearly observed at the other distances downstream. The amplitude of the enhancements relative to ambient are similar to within 20 $30 \%$ at the same values of $r / R_{0}$ at both pressures.

Recently, Fournier and Pigache ${ }^{7}$ have observed the influence of slow ions on ion current structure in the wake region at system pressures of $\sim 10^{-7}$ Torr in the ONERA facility. They concluded that the influence of slow ions on ion current structure is negligible at this pressure. On the basis of the data taken in both the MSFC and ONERA facilities, we conclude that when $\alpha \leq 0.3$ the slow ions have only a minor effect on the ion current structure observed in the wake region.

In Fig. 3 we show profiles of electron current taken downstream behind the sphere at $r / R_{0}=7.2$ and at conditions identical to those in Fig. 2. The position $r / R_{0}=7.2$ is close to a Mach number of radii downstream from the sphere center. This particular position was chosen since finding a current enhancement in this vicinity may have a bearing upon the validity of using a fluidlike or a kinetic approach in treating this type of flow problem. ${ }^{11}$

The electron current was determined from the inflection point of a current-voltage curve taken with a Langmuir probe. It should be noticed that at the higher pressure the enhancement has been so reduced that it is virtually undetectable. This is in contrast with the results of Fig. 2 where an ion current enhancement at $r / R_{0}=7.2$ is visible at both pressures. It follows that, at the higher pressure of $2.5 \times 10^{-5}$ Torr, the electron current observations are influenced by the presence of slow ions.

Figure 4 shows a representative series of transverse $T_{c}$ profiles measured at distance or $r / R_{0}=1.25$ downstream of 


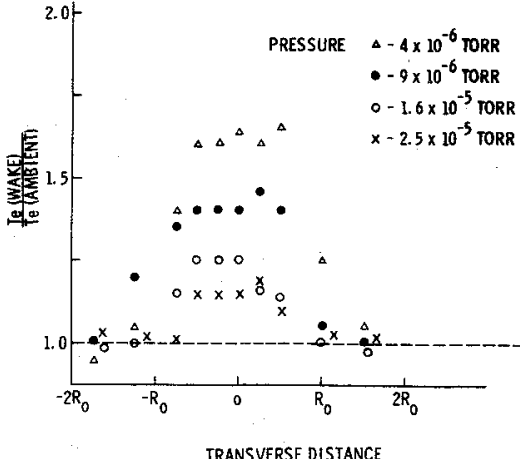

Fig. 4 Transverse electron temperature profiles (normalized to the ambient $T_{e}$ ) taken at $1.3 R_{0}$ downstream from the sphere center (radius $=R_{\theta}=4 \mathrm{~cm}$ ) at several system pressures and for flow conditions $E_{i} \sim 4.5 \mathrm{eV}, T_{e} \sim 800 \mathrm{~K}, n \sim 5 \times 10^{4} / \mathrm{cm}^{3}$, and $\phi_{s} \sim 0.3 \mathrm{~V}$.

the sphere in the pressure range $4 \times 10^{-6} \leq P \leq 2.5 \times 10^{-5}$ Torr. The conditions are identical to those of Fig. 2 except for $\phi_{s}$ which is now equal to $+0.3 \mathrm{~V}$. We have chosen to examine the influence of the slow ions on the electron temperature at the closest vicinity to the sphere downstream, since it is in this region that the effect of $T_{e}$ (wake) $>T_{e}$ (ambient) is pronounced. ${ }^{12-14}$

As is seen from Fig. 4, $T_{e}$ (wake) is larger than $T_{e}$ (ambient) for pressures smaller than about $1.6 \times 10^{-5}$ Torr. For the higher pressures, the enhancement in $T_{e}$ (wake) with respect to $T_{c}$ (ambient) is close to the experimental error in the electron temperature determination. The trend seems, however, to be clear. Namely, as the pressure increases the influence of the slow ions on the electron temperature observations is more significant, which is similar to the influence of the slow ions on the electron current profiles (see Fig. 3).

In summary, the findings of this study indicate that the effect of slow ions, when $\alpha \leq 0.3$, is not significant for measurements of ion current variations in the wake zone. However, this is not the case when studies are aimed at the quantitative examination of electron current and temperature variations in the near wake zone. In these instances, the measurements of electron properties in the wake should be done at very low systems pressures or over a range of system pressures in order to ascertain the effect of slow ions.

\section{References}

${ }^{1}$ Liu, V. C., "Ionospheric Gas Dynamics of Satellites and Diagnostics Probes," Space Science Reviews, Vol. 9, 1969, p. 423.

${ }^{2}$ Gurevich, A. V., Pitaevskii, L. P., and Smimova, V. V., "Ionspheric Aerodynamics," Soviet Physics-USPEKHI, Vol. 12, 1970, p. 505 .

${ }^{3}$ Liu, V. C., "On Ionospheric Aerodynamics," Progress in Aeronautical Sciences, Vol. 16, 1975, p. 273.

${ }^{4}$ Samir, U. and Wrenn, G., "Charged Particle Distribution in the Nearest Vicinity of Ionospheric Satellites: Comparison to the Main Results from the Aeriel I, Explorer 31 and Gemini/Agena 10 Spacecraft," Particle and Photon Interactions with Surfaces in Space, (ed. G. Grard), North Holland, Amsterdam, 1973, p. 15.

${ }^{5}$ Samir, U., "Space-Related Laboratory Simulation Studies Plasma Flows Over Bodies in Space," Transactions American Geophysical Union, EOS, Vol. 56(12), 1975, p. 1039.

${ }^{6}$ Oran, W. A., Stone, N. H., and Samir, U., "Parametric Study of Near-Wake Structure of Spherical and Cylindrical Bodies in the Laboratory," Planetary and Space Science, Vol. 22, 1974, p. 379.

${ }^{7}$ Fournier, G. and Pigache, D., "Wakes in Collisionless Plasmas," Physics of Fluids, Vol. 18(9), 1975, p. 1443.

${ }^{8}$ Hester, S. D. and Sonin, A. A., "A Laboratory Study of the Wakes of lonospheric Satellites," AIAA Journal, Vol. 8, June 1970 , pp. 1090-1098.

${ }^{9}$ Martin, A. R. and Cox, R. N., "Simulation of a Spacecraft Interaction with a Parallel Streaming Plasma," presented at the NASA/ASTN/LES, 7th Space Simulation Conference, 1974.

${ }^{10}$ Pigache, D., "A Laboratory Simulation of the Ionospheric Plasma," AIAA Paper 71-608, Palo Alto, Calif., 1971.
${ }^{11}$ Samir, U., Stone, N. H., and Oran, W. A., "Does a Two Stream Flow Model Apply to Wakes of Large Bodies in Space," Astrophysics and Space Sciences, Vol. 31, 1974, p. L1.

${ }^{12}$ Samir, U. and Wrenn, G. L., "Experimental Evidence of an Electron Temperature Enhancement in the Wake of an Ionospheric Satellite," Planetary and Space Science, Vol. 20, 1972, p. 899.

${ }^{13}$ Troy, B. E. Jr., Maier, E. J., and Samir, U., "Electron Temperature in the Wake of an Ionospheric Satellite," Journal of Geophysical Research, Vol. 80, 1975, p. 933.

${ }^{14}$ Oran, W. A., Samir, U., Stone, N. H., and Fontheim, E. G., "Laboratory Observations of Electron Temperature in the Wake of a Sphere in a Streaming Plasma," Planetary and Space Science, Vol. 23, 1975, p. 1081.

\section{Aft-Body Drag Reduction by Combined Boat-Tailing and Base Blowing at $M=3$}

\author{
Robert H. Korkegi* and L. Michael Freeman $\dagger$ \\ Air Force Aero Propulsion Laboratory \\ Wright-Patterson Air Force Base, Ohio
}

\section{Introduction}

$\mathbf{T}$ HE major source of drag of a blunt-based slender body at moderate supersonic speeds is the aft-body. The primary factor accounting for high aft-body drag is the low base pressure. Two potentially effective means of increasing base pressure are boat-tailing and small rates of gas injection at the base. A recent review of the base flow problem including injection ${ }^{1}$ presents several semiempirical methods of calculating base pressure but points out that the greatest uncertainities in the flow are associated with the base corner region and development of the mixing layer, to which the axisymmetric geometry adds further complication.

The effect of boat-tailing is well covered in an earlier review ${ }^{2}$ which presents evidence that conical boat-tails are superior to other shapes (ogival, concave) and that appreciable reduction in base drag can be obtained with boat tails of moderate angles and lengths up to about 1 or 1.5 calibers. An early study of base bleed to reduce supersonic aft-body $\mathrm{drag}^{3}$ showed that small rates of injection caused the base pressure to rise, but increased rates led to a reversal attributed to entrainment of the base flow by the bleed air due to its increasing velocity. At very low injection rates, of course, the opposite is true-the flow entrains the low momentum gas. Factors which influence the effectiveness of base injection, extensively studied by Bowman and Clayden, ${ }^{4-7}$ include: the gas injection geometry, the temperature of the injected gas, its molecular weight, and the boundary-layer thickness.

The present study is concerned with the combined use of boat-tailing and base blowing to minimize the aft-body drag of a projectile configuration at Mach 3 and high Reynold's numbers. A more extensive description of the study and discussion of the flowfield is given in Ref. 8 .

\section{Test Apparatus}

The ogive-cylinder projectile model of $30 \mathrm{~mm}$ ( 1.181 in.) diameter with rifling ring and interchangeable aft-bodies shown in Fig. 1 was tested in the Air Force Flight Dynamics Laboratory $8 \times 8$ in. Mach 3 high Reynolds number wind tunnel. Air and helium were the two gases injected through

Received March 1, 1976; revision received April 26, 1976.

Index categories: Jets, Wakes, and Viscid-Inviscid Flow Interactions; Supersonic and Hypersonic Flow.

*Senior Scientist, Ramjet Division. Associate Fellow AIAA.

$\dagger$ Aerospace Development Engineer, Aeromechanics Division, Flight Dynamics Laboratory, Wright-Patterson Air Force Base, Ohio. Captain, U.S. Air Force. 